\title{
A STEP TOWARD THE ELUCIDATION OF QUANTITATIVE LAWS OF NATURE
}

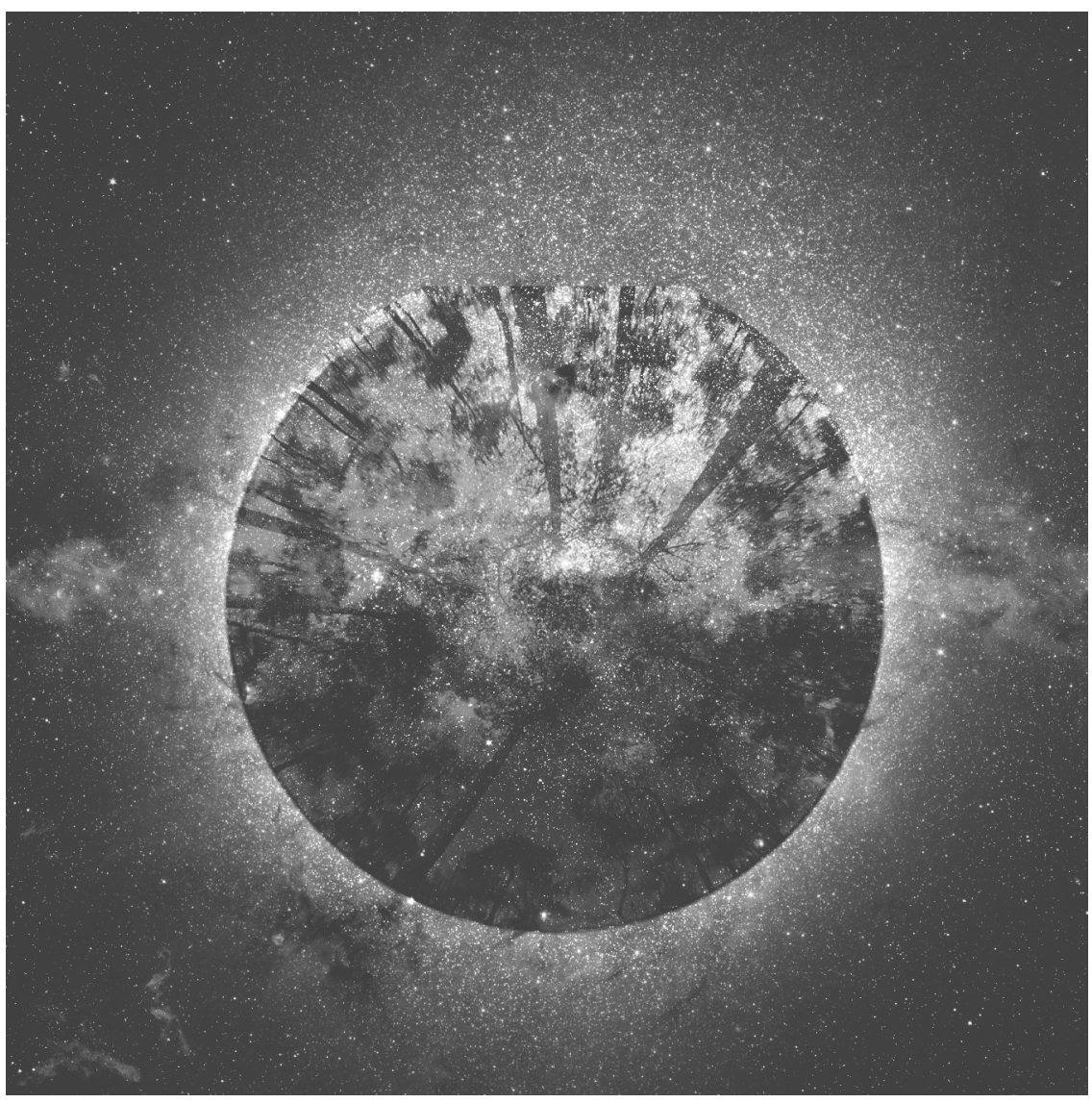

STEPHEN PERRY 


\section{ABSTRACT}

When we mathematically model natural phenomena, there is an assumption concerning how the mathematics relates to the actual phenomenon in question. This assumption is that mathematics represents the world by "mapping on" to it. I argue that this assumption of mapping, or correspondence between mathematics and natural phenomena, breaks down when we ignore the fine grain of our physical concepts. I show that this is a source of trouble for the mapping account of applied mathematics, using the case of Prandtl's Boundary Layer solution to the Navier-Stokes equations. 


\section{CONSIDERATIONS OF THE INTERFACE BETWEEN MATHEMATICS AND THE SCIENCES}

It is widely accepted that mathematics is of great use to science. Mathematics seems to give our laws of nature more detail and enhance our ability to make predictions. We see this when scientists try to predict natural phenomena-from the equations of celestial mechanics they develop to precisely calculate the motions of the planets, to the current complex system of equations they use to describe the global climate system. What is curious about this is why mathematics is so useful in the natural sciences. This sentiment is encapsulated in Eugene P. Wigner's landmark paper, "The Unreasonable Effectiveness of Mathematics in the Natural Sciences," in which Wigner wonders at the mysterious ways that mathematics provides laws of nature with their precision and predictive utility. ${ }^{1}$ How exactly mathematics provides this is unclear, especially given its abstract nature-as opposed to the decidedly non-abstract subject matter of the natural sciences. As Bertrand Russell stated, "It must have required many ages to discover that a brace of pheasants and a couple of days are both instances of the number two." 2 The question is how an abstract concept such as "one" — which seems to lack the character of concrete "physical world" concepts such as "rock" — could possibly aid us in obtaining knowledge about the physical world.

One of the main approaches to explaining how mathematics can aid in representing the physical world is commonly known as the "mapping account." In short, the mapping account asserts that the structure generated by a mathematical representation is in some specific sense-usually defined in terms of the mathematical notions of isomorphism and homomorphism-similar to the structure of the physical system or phenomenon being represented. ${ }^{3}$ This account has some intuitive appeal; consider a problem where we are told that Angeline has 3 apples and Keisha has 5 apples and are then asked how many apples Angeline and Keisha have together. Clearly, the number " 3 " corresponds in some way to "how many apples Angeline has," the number " 5 " corresponds in some way to "how many apples Keisha has," and the operation of addition corresponds in some way

1 Eugene P. Wigner, "The Unreasonable Effectiveness of Mathematics in the Natural Sciences," Communications on Pure and Applied Mathematics 13, no. 1 (1960), 10.1002/cpa.3160130102.

2 Bertrand Russell, Introduction to Mathematical Philosophy (New York: Macmillan, 1920).

3 See Christopher Pincock, "A Role for Mathematics in the Physical Sciences," Noûs 41, no. 2 (2007), 10.1111/j.1468-0068.2007.00646.x. The mapping account has had many recent formulations, notably by Pincock. 
to the "and" in "how many apples Angeline and Keisha have together." Thus, the problem can be solved using the mathematical equation 3 $+5=8$. The structure of the mathematics corresponds, or "maps," to the structure of the system under consideration, in this case on an element-by-element basis.

However, mathematical representations of the physical world quickly become more complicated. In the two-body problem, we consider two bodies moving in a vacuum and attempt to find and solve the dynamical equations expressing their motion, given the force of gravity acting between them. This is a relatively simple problem that most undergraduate physics students could solve with little trouble. We end up with a single differential equation that is only required to express motion in two dimensions, which produces one line of force. However, as soon as we add another body to this system - the "threebody problem" - it becomes exponentially harder to find solutions. There are three lines of force, and they interact with one another in ways that are hard to isolate. We end up with a system of several differential equations, and an exact solution is difficult to find.

Application of the mapping account becomes less and less intuitive as the mathematics used to represent a physical system become not only more complicated, but also begin to employ idealizations. Idealizations occur when our representation utilizes an assumption that we know to be false, such as assuming an infinitely deep ocean when constructing a representation for water wave dispersion. If our mathematics involves false assumptions about the world, then idealizations trouble the idea that our mathematics can map directly onto the natural world.

The mapping account of applied mathematics has faced many objections regarding both the role mathematics plays in explanation and how it can apply in situations where mathematical representations are inconsistent. ${ }^{4}$ In this paper, I argue that a further obstacle for the mapping account is the view it employs of physical concepts. This problem with the mapping account traces back to our intuitions about how mathematics is applied, and how scientific laws work in

4 See Robert Batterman, "On the Explanatory Role of Mathematics in Empirical Science," The British Journal for the Philosophy of Science 61, no. 1 (2010), 10.1093/bjps/axp018. Batterman critiques the mapping account's explanatory promise. See Pincock's reply in Christopher Pincock, "On Batterman's 'On the Explanatory Role of Mathematics in Empirical Science," The British Journal for the Philosophy of Science 62, no. 1 (2011), 10.1093/bjps/axq025. Additionally, a recent critique of the mapping account has been given in Colin McCullough-Benner, "Representing the World with Inconsistent Mathematics," The British Journal for the Philosophy of Science (2019), 10.1093/bjps/axz001. 
general. To flesh out this idea, I consider a particularly troublesome application of mathematics: Prandtl's Boundary Layer solution to the Navier-Stokes equation for fluid flow bordering a solid. The problem with Prandtl's Boundary Layer solution is that we end up with two distinct systems of equations to describe what we think of as a single fluid. This should trouble us if we tap into our intuitions about how mathematics is applied, especially as encoded in the mapping account.

A good preliminary question is why we should care about Prandtl's Boundary solution. After all, it works and nothing seems to be "wrong" with it, per se. However, a problem does lie in the way this solution's success grates against our conception of how scientific theories function. There does not seem to be any physical counterpart to the mathematical "boundary layer" posited by Prandtl's solution, and it is unclear why we are licensed to use different approaches to represent different patches of the fluid.

Navier-Stokes' intractability itself is puzzling. Physicists claim that the Navier-Stokes equation gives the most accurate representation of fluid flow. However, we can only show that it works in special circumstances, or by using numerical approximation of the solution. ${ }^{5}$ Consider an example simpler than Navier-Stokes equation: the kinematic equations of classical mechanics. These equations relate the position, velocity, and acceleration of an object moving with a constant acceleration. We can check that the equations "work" by constructing an experiment with certain initial conditions, solving the kinematic equations for certain values given the initial conditions, and checking how these calculated values match with what we actually measure. We cannot do this with the Navier-Stokes equations due to their intractability. How, then, do we "know" that Navier-Stokes provides an accurate description of fluid flow, if we cannot "prove" it in the same way we would for simpler equations?

\section{PHYSICAL CONCEPTS AND MATHEMATICAL REPRESENTATION}

There are several approaches to diagnose the perceived problem with Prandtl's Boundary Layer solution. The simplest approach would be to throw up our hands and cry, "It's an approximation!" However, this seems like an unsatisfactory approach of giving up; it avoids the question of why this seemingly counterintuitive application of

5 Numerical approximation is a technique for approaching differential equations which does not attempt to find an exact solution, but rather an approximate one by taking small steps in values. Today, it is usually done via computer simulation. 
mathematics works by appealing to a "close enough" sort of response. We now have to ask why the approximation works so well. We want to know to what end we are justified in using it and where it may break down. These equations are used in significant circumstances such as airplane wing design and climate modeling, so it is important to know where they may not accurately represent. We must develop a more nuanced approach.

On a mapping account analysis of the situation, the problem is quite acute. A single natural system is represented by two different systems of equations, so the mapping account asserts that each set of equations is structurally similar in a specific way to some part of the fluid flow. However, this implies that the fluid flow has two separate structures, although they are related along their boundary. This result seems quite counterintuitive-clearly we took a misstep somewhere along the way. But where? As explained by Pincock, the mapping account posits three things: (i) there is a structure generated by the mathematics, (ii) there is a structure associated with the physical system under consideration, and (iii) there exists a specific kind of correspondence between the two structures. ${ }^{6}$ We have little reason to doubt (i). We may want to question (ii), depending on if we are scientific realists. ${ }^{7}$ However, further reflection shows that structure in nature does not have to be the exact structure posited by our theory. The mapping account makes no commitment to scientific realism; it simply tries to explain why our theory is successful. So, our last option is to interrogate the core of the mapping account: the correspondence asserted between the two structures.

The correspondence claim asserts that we can capture the significant aspects of the natural system with mathematics. This is exactly what Wigner questions. How can we capture aspects of the physical world with mathematics when we consider concepts that go beyond counting discrete objects? This is where measurement comes into play. We can measure several fairly simple concepts in a

6 I will note here that this is not quite true. Pincock specifically posits an isomorphism or homomorphism between the two structures; this is the "special" part of the correspondence between the structures that I do not have time to fully dive into here. Thus, there are actually two parts to (iii): (iii ${ }_{1}$ ) there exists a correspondence between the possible occupants of the two structures, and ( $\left.\mathrm{iii}_{2}\right)$ this correspondence preserves a relation that "only occupants $\mathrm{x}, \mathrm{y}$, and z can exist in combination." However, considerations of (iii) and ( iii $_{2}$ ) in combination will eventually lead us to the line of questioning I pursue here.

7 Generally, scientific realism is the view that our best scientific theories accurately describe "what is out there." In other words, a scientific realist believes that the entities posited to exist by our scientific theories do, in fact, exist. 
straightforward way. We measure length by having a unit length (e.g. an inch or a mile) and counting how many unit lengths constitute a given length. This measurement exhausts what we need to know about length, given our conceptualization of length. Likewise, we measure time by having a unit time (e.g. an hour or a year) and counting how many unit times constitute a given period. It is quite similar to the length concept, as is demonstrated by the phrase "length of time" that is often used to describe the passage of time. It can certainly be argued at this point that matters of precision make these measurement processes significantly less certain (e.g. measuring microscopic or astronomical lengths). But barring precision, it seems relatively straightforward that these measurements exhaust their respective concepts.

Hasok Chang's discussion of the history of thermometry demonstrates many obstacles in formulating a measurement process for physical concepts more complex than length, such as temperature, force, and viscosity. ${ }^{8}$ Chang begins by discussing what he calls "thermoscopes," which consisted of a wide variety of devices showing change in temperature without giving a numerical scale or standard. These thermoscopes relied on the fact that fluids expand as they get hotter, and they constrained the motion of the fluid's expansion along a line. In other words, these devices created an association between temperature and length. But when the time came to standardize thermoscopes, there were many different suggestions as to what fixed points of the scale should be used to calibrate the thermoscopes. Even after an agreement was reached, there was still doubt as to whether the "standard" thermometer did, in fact, track the linear increase of temperature. After all, there was no guarantee that a linear expansion of the fluid corresponded with a linear increase in temperature. Further, the thermometers consisting of a fluid in a tube failed to function in extreme cold or extreme heat. We can ask whether this measurement process exhausts our temperature concept. If we accept that the temperature concept is about the motion of individual molecules, we might doubt that our measurement process actually exhausts the temperature concept. After all, the temperature measurement process occurs on a macroscopic scale, so how could it track this microscopic process? The answer is that it does not. It simply averages to some degree that we assume to be "good enough," depending on the scale at which we are working.

Similar questions arise when we try to analyze the viscosity concept at work in the Navier-Stokes equation. Viscosity-as it was

8 Hasok Chang, Inventing Temperature: Measurement and Scientific Progress (New York: Oxford University Press, 2007). 
understood in the derivation of Navier-Stokes and still is todayinvolves forces between individual molecules of the fluid which inhibit fluid motion. Viscosity was often described as "internal fluid friction" or "fluid resistance." In other words, our viscosity concept tries to capture the various processes responsible for a fluid's resistance to movement. However, in the Navier-Stokes equation, viscosity is reduced to a single number, a characteristic constant determined by the fluid under consideration, analogous to density or melting point. As we did with the temperature concept, we may wonder whether this measurement process — ascribing a single viscosity constant to a given fluid - exhausts our concept of viscosity, or internal fluid friction. It seems there is more going on within the viscosity concept than can be captured by a single number. Let us go into more detail about NavierStokes in order to tease out what is going on here.

\section{THE NAVIER-STOKES EQUATION AND VISCOSITY}

The Navier-Stokes equation is regarded as the fundamental equation of fluid mechanics. As it is understood today, it attempts to describe fluid motion by considering an infinitesimal volume of fluid. The forces exerted on the volume of fluid are equated with the product of the volume's acceleration and the density of the fluid. Incompressibility is then assumed, yielding the following equations:

1. $\mu \Delta v-\nabla P-\rho f(x, t)=\rho(\partial v / \partial \dagger+v \cdot \nabla v)$

2. $\nabla \cdot v=0^{9}$

The Navier-Stokes equations are notoriously intractable in terms of finding exact solutions. The trouble mostly comes from the secondorder partial derivative that occurs in the viscosity term. Earlier hydrodynamics equations from Euler essentially ignore viscosity, but otherwise mirror Navier-Stokes. However, the Euler equations yield

9 Here, $\rho$ is the density of the fluid, $v$ is the velocity vector of the volume of fluid, $\mu$ is the viscosity of the fluid, $P$ is the pressure velocity field acting on the fluid, $f$ is the sum of external forces acting on the fluid at a specific position and time, and $t$ is time. Equation (1) is analogous to Newton's Second Law, F=ma. Starting on the right-hand side, the density (mass per unit volume) stands in for the mass of the infinitesimal volume of fluid under consideration, and it is multiplied by the acceleration of this volume of fluid. Then, on the left-hand side, we see the sum of the forces acting on the volume of fluid. The first term represents force due to the fluid's viscosity, the second term represents the force of pressure, and the last term represents external forces per unit volume. Equation (2) expresses the incompressibility constraint, which essentially encodes the conservation of mass. Along with particular boundary conditions, this equation yields the most accurate analytic description of fluid flow. 
counterintuitive results regarding fluid flow near solids, so they are quite useless for practical purposes. ${ }^{10}$

Various simplifications have been proposed for particular cases of fluid flow, notably Prandtl's Boundary Layer solution for fluid flow bordering a solid. Prandtl's solution posits a very thin "boundary layer" right where fluid flow meets the solid (say, an immersed object or the walls of a pipe). Prandtl derived "boundary layer equations" that describe fluid flow inside this boundary layer - tractable equations which still recognize the importance the viscosity of the fluid comes to have near the solid. The result of adding this second set of equations is that we effectively divide fluid flow into the boundary layer-where fluid flow is described by the boundary layer set of equations - and the rest of the fluid - where fluid flow is described by the Eulerian hydrodynamics equations. This is the part of Prandtl's solution that makes a mapping account interpretation counterintuitive; we have divided the fluid flow into two regions and performed different mathematical manipulations to Navier-Stokes for each region. The key difference between the two equations and the difficult concept leading to Prantl's solution is viscosity. So, we will further probe into the viscosity concept here.

\section{CONCLUSION: THE FINE GRAIN OF CONCEPTS}

In his work on concepts, Mark Wilson emphasizes the "fine grain" of concepts that is often lost in our use of them. ${ }^{11}$ While our use of a concept may be successful for some time, we eventually run into trouble with its application, as we do with viscosity. Suddenly, the fine grain matters quite a bit more. For viscosity, this can be seen in the addition of Prandtl's Boundary Layer solution for fluid flow bordering an object, where the fine grain of the viscosity concept becomes significantly more important. The behavior that viscosity attempts to capture varies greatly depending on the distance of the portion of fluid we are considering from bordering solids. However, instead of trying to capture everything at work here, physicists do something Wilson calls "physics avoidance;" they compress the complex interaction going on to a singularity (the boundary layer edge), obtain approximate equations for the fluid's

10 See Olivier Darrigol, "Between Hydrodynamics and Elasticity Theory: The First Five Births of the Navier-Stokes Equation," Archive for History of Exact Sciences 56, no. 2 (2002); and Olivier Darrigol, Worlds of Flow: A history of hydrodynamics from the Bernoullis to Prandtl (New York: Oxford University Press, 2005) for more on the history of hydrodynamics and Navier-Stokes.

11 Mark Wilson, Wandering Significance: An Essay on Conceptual Behavior (New York: Oxford University Press, 2006). 
behavior on either side of this singularity, and match the equations at the singularity. In other words, they do their best to avoid addressing the fine grain of the viscosity concept, since mathematically representing that fine grain turns out to be incredibly complex.

There are many instances of this "physics avoidance," even in classical mechanics. Wilson's main example is the collision of billiard balls. Any beginning physics student learns that conservation of momentum for perfectly elastic solids can model a billiard ball collision, and this is a relatively easy problem to solve mathematically. However, there is more going on in the actual billiard ball collision than is represented in the conservation of momentum. The balls deform in ways that are difficult to model, and shock waves propagate through each ball. This fine grain of collision becomes increasingly significant as we consider collisions involving higher speeds and different types of objects. For example, when we want to consider collisions of water balloons, the deformations upon collision become very important. So, we have another instance where a concept has a fine grain that we tend to ignore when possible.

The problem with the mapping account and its guiding intuitions is that it does not recognize this fine grain of concepts when the equations we use also ignore it. This is why a mapping account analysis of the Navier-Stokes/Prandtl situation seems so problematic; the concepts encoded in the equations do not actually map to the more nuanced details of the physical world. Furthermore, this implies that our intuitions about mathematically describing natural phenomena deserve a closer look, as this fine grain often only shows up when our representations fail to be accurate. A careful approach to these issues should involve not only a philosophical analysis of the concepts both in theory and in use, but also a historical analysis of concepts and mathematical representations. Together, these analyses can illuminate aspects of concepts that are not recognized in contemporary scientific practice and philosophy of science. 


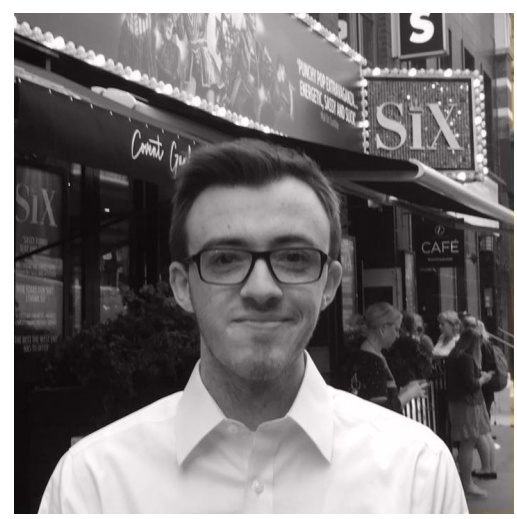

Stephen Perry will graduate from the University of Kentucky in Lexington, Kentucky in 2021 with a double major in Philosophy and Mathematics, and minors in Physics, Cognitive Science, and Theatre. His main interests are history and philosophy of science and mathematics, science and society, fluid mechanics, epistemology, and phenomenology. 
